\section{AB0359 ASSESSING DEPRESSION IN PATIENTS WITH ESTABLISHED RHEUMATOID ARTHRITIS-HOW DO DIFFERENT APPROACHES COMPARE?}

S.L. Hider ${ }^{1}, 2$, S. Ryan ${ }^{1}$, N. Dale ${ }^{1}$, N. Stanyer ${ }^{1}$, A. Machin ${ }^{2}$,

C.A. Chew-Graham ${ }^{2,3}$. ${ }^{1}$ Haywood Academic Rheumatology Group, Haywood Hospital, Staffordshire; ${ }^{2}$ Research Institute Primary Care and Health Sciences, Keele University, Keele; ${ }^{3}$ Collaboration for Leadership in Applied Health Research and Care (CLARHC), West Midlands, United Kingdom

Background: Depression is common in patients with rheumatoid arthritis and negatively impacts on their quality of life and disease outcomes, including disease activity and treatment response. Several case-finding tools for depression are available, including the PHQ2, which is both valid (1) and easy to use.

Objectives: The aim of this study was to compare the prevalence of depression using case finding tools and self-report measures in patients with established RA. Methods: Patients with established RA, attending a nurse-led annual review clinic, which aimed to offer patients a holistic review, were asked to complete a short questionnaire including demographics and self-reported comorbidity. The presence of depression was assessed in 3 ways a) PHQ2 score $\geq 3$ b) Self recorded "ever" depression using the self-administered report comorbidity questionnaire (2) and c) Self report health status using the EQ5D - which includes a statement regarding current anxiety/depression (dichotomised into no anxiety/depression vs. slight/moderate/severe/extremely anxious or depressed. Ethical approval was obtained (15-WS-0063).

Results: 179 RA patients provided data. Of these $119(66 \%)$ were female and the mean (sd) age was $67.1(11.7)$ years. 59 patients $(33 \%)$ reported they had ever had depression using the self-report comorbidity questionnaire and $25(14 \%)$ indicated they were currently receiving treatment. $68(38 \%)$ indicated they were currently slightly (or more) anxious or depressed when assessed with the EQ5D. $37(21 \%)$ scored positively on the PHQ2. There was good concordance between the PHQ2 and EQ5D at higher levels of depression, in that all those with severe or extreme anxiety or depression on EQ5D also scored positively on the PHQ2. However, of those with moderate anxiety/depression on EQ5D, 4/14 patients scored less than 3 using the PHQ2 score.

Conclusions: Depression is common in patients with established RA. Use of the PHQ2 case-finding questions in patients with established RA, may help clinicians identify patients who may benefit from more detailed assessment of mood and interventions to improve their outcomes. Reliance should not be placed on a single tool, and exploration of mood should be part of routine assessment of a patient with RA.

References:

[1] Meader N,et al. BJGP 2011:61: 733-734.

[2] Sangha O, et al.Arthritis Rheum 2003;49:156-63.

Acknowledgements: ND was funded by the Haywood Foundation. CCG is funded by the National Institute for Health Research (NIHR) Collaborations for Leadership in Applied Health Research and Care West Midlands. The views expressed are those of the authors and not necessarily those of the NHS, NIHR or the Department of Health.

Disclosure of Interest: None declared

DOI: 10.1136/annrheumdis-2017-eular.5996

\section{AB0360 CLINICAL AND HIGH RESOLUTION CT STUDY OF THE LUNGS IN 96 EGYPTIAN PATIENTS WITH RHEUMATOID ARTHRITIS}

S. Tharwat, D. Shahin, A. Abdesalam, A.F. Barakat. Internal Medicine (Rheumatology \&/mmunology), Mansoura University Hospital, Mansoura, Egypt

Background: Lung involvement is a common extra-articular manifestation of rheumatoid arthritis (RA) that confers significant morbidity and mortality. However, this issue has not been sufficiently studied in Egyptian patients.

Objectives: To assess the prevalence of pulmonary abnormalities by high resolution computed tomography (HRCT) chest in RA patients and their association with clinical variables.

Methods: Ninty-six patients with RA were assessed regarding their age, gender,

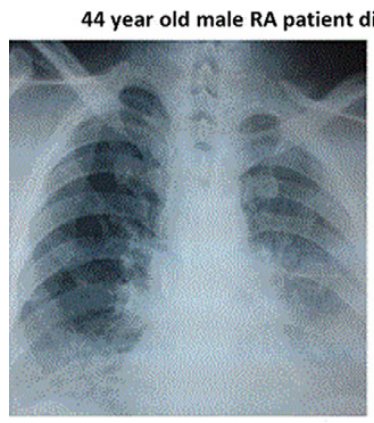

Chest $x$-ray shows reduced

lung volumes with

bilateral reticular opacities duration of RA disease, sicca symptoms, presence of subcutaneous rheumatoid nodules, rheumatoid factor, respiratory symptoms, use of medications, disease activity of RA using Disease Activity Score 28 (DAS28), basic laboratory investigations and pulmonary abnormalities in plain x-ray and HRCT.

Results: HRCT abnormalities were identified in $25 \%$ of the patients; $58.3 \%$ had respiratory symptoms while $41.7 \%$ were subclinical. Dyspnea and cough were the most frequent symptoms. HRCT abnormalities were interstitial lung disease (ILD) in 11 patients $(11.5 \%)$, pleural involvement in 8 patients $(8.3 \%)$, consolidation in 4 patients $(4.2 \%)$, diffuse alveolar hemorrhage in 3 patients $(3.1 \%)$, bronchiectasis in 2 patients $(2.1 \%)$, and apical fibrosis with cavitation in only 1 patient $(1 \%)$. Patients with dyspnea, cough, chest rales, cutaneous rheumatoid nodules, and who received disease modifying antirheumatic drugs (DMARDs) combination were more likely to have HRCT abnormalities.

Conclusions: Pulmonary involvement is not uncommon in RA patients, and HRCT is a sensitive tool in pulmonary evaluation.

Disclosure of Interest: None declared

DOI: 10.1136/annrheumdis-2017-eular.5037

\section{AB0361 BODY COMPOSITION AND PHYSICAL ACTIVITY IN PATIENTS WITH RHEUMATOID ARTHRITIS (AR)}

S. Manrique-Arija, N. Mena-Vazquez, M. Rojas-Giménez, C. Fuego,

S. Abad-Sanchez, I. Ureña-Garnica, C. Domic, F.G. Jiménez-Núñez,

M.C. Ordoñez-Cañizares, R. Caparros-Ruiz, L. Cano-Garcia, A. Belmonte,

B. Panero-Lamothe, A. Fernandez-Nebro. Department of Rheumatology at the

University Regional Hospital of Malaga (HRUM). Institute for Biomedical

Research in Malaga (IBIMA). Malaga University., Malaga, Spain

Objectives: To describe the body composition and physical activity of patients with RA

Methods: Design: Observational case-control study. Population: 31 consecutive RA-patients (ACR/EULAR 2010 criteria) selected from a prospective inception cohort (diagnosis of RA between 2005 and 2012), and 31 sex and age-matched voluntary controls. Protocol: We included 62 subjects who were assessed using dual-body X-ray absorptiometry (DEXA) for the assessment of body composition at study entry. Data on clinical activity were also collected from the onset of the disease.The level of physical activity was assessed according to the abbreviated protocol of the International Physical Activity Questionnaire (IPAQ). Main outcome: Fat mass index (FMI) and fat free mass index (FFMI).FMI was defined as fat mass $(\mathrm{kg}) /$ height $\left(\mathrm{m}^{2}\right)$ and FFMl as lean mass $(\mathrm{kg}) /$ height $\left(\mathrm{m}^{2}\right)$. Secondary outcome: description of the anthropometric parameters:BMI (body mass index),waist-hip index. Variables: Demographic, treatment, clinical-analytical variables: Disease Activity Score of 28 joints (DAS28-ESR), Clinical Disease Activity Index (CDAI) and Simplified Disease Activity Index (SDAI);Anti-cyclic citrullinated peptide (ACPA), Rheumatoid factor (RF), Health Assessment Questionnaire (HAQ)and erosions.BMI was categorized according to the OMS classification. Waist- hip index (central obesity $>1$ for men and $>0.8$ for women). Statistical analysis: Descriptive and paired T-test or ranks test and Wilcoxon signed rank, as required Results: We included 62 subjects,31 RA (50\%) and 31 controls (50\%). The mean duration of the disease of RA patients was 84.4 months. With a DAS28 mean of $3.04(0.8)$, mean HAQ of $0.77(0.6)$. The majority presented erosions $(64.5 \%)$ RF $(83.9 \%)$ and ACPA (77.4\%) positive. Differences in clinical and densitometric anthropometric parameters between cases and controls are shown in Table 1. Significant differences were observed in the proportion of overweight subjects between cases and controls $(p=0.03)$. A higher percentage of controls had moderate to high physical activity compared with the patients $(65.5 \%$ vs $34.8 \%)$. The majority of patients with RApatients had low physical activity (74.2\%). These had a mean (SD) of $\mathrm{HAQ}$ higher than patients with moderate-high physical activity [1.2 (0.9) vs $0.66(0.6) ; p=0.09]$. Finally, patients performing moderate-high physical activity had less inflammatory activity than those performing low physical activity, with a DAS28 mean of $2.69(0.7)$ vs $3.08(0.9) ; p=0.308$, respectively

\begin{tabular}{lccc}
\hline Variable & Cases $(\mathrm{n}=31)$ & Controls $(\mathrm{n}=31)$ & $\mathrm{P}$ \\
\hline Age (years), mean (SD) & $53.6(12.1)$ & $53.6(12.3)$ & 0.999 \\
Sex (Female), $\mathrm{n}(\%)$ & $27(87)$ & $27(87)$ & 1 \\
Body mass index (BMI), mean (SD) $>25 \mathrm{n}(\%)$ & $25(80.6)$ & $17(54.8)$ & 0.03 \\
Waist perimeter (cm), mean (SD) & $88.7(13.2)$ & $89.1(14.4)$ & 0.891 \\
Hip perimeter (cm), mean (SD) & $103.9(9.5)$ & $106.8(15.8)$ & 0.395 \\
Waist-hip index, mean (SD) & $0.85(0.1)$ & $0.84(0.1)$ & 0.653 \\
- Total fat (kg), mean (SD) & $28.2(9.3)$ & $29.1(10.9)$ & 0.737 \\
- Total lean (kg), mean (SD) & $39.5(7.6)$ & $38.2(9.6)$ & 0.555 \\
- Total mass (kg), mean (SD) & $70.08(13.8)$ & $69.2(15.5)$ & 0.817 \\
FMl (\%) median (p25-p75) & $10.44(8.3-12.4)$ & $9.51(8.2-13.8)$ & 0.882 \\
FFMl (\%) median (p25-p75) & $14.27(13.1-15.8)$ & $13.83(13.1-15.7)$ & 0.522 \\
\hline
\end{tabular}

Conclusions: Patients with RA compared to controls show more overweight and less physical activity

Disclosure of Interest: None declared

DOI: 10.1136/annrheumdis-2017-eular.6056 